\title{
Tumor vaccination after allogeneic bone marrow cell reconstitution of the non-myeloablatively conditioned tumor-bearing murine host \\ M Zöller*
}

Address: Department of Tumor Progression and Tumor Defense, German Cancer Research Center, D-69120 Heidelberg, Germany

Email: M Zöller* - m.zoeller@dkfz.de

* Corresponding author

from Association for Immunotherapy of Cancer: Cancer Immunotherapy - $2^{\text {nd }}$ Annual Meeting

Mainz, Germany, 6-7 May 2004

Published: I July 2004

Received: 28 April 2004

Cancer Cell International 2004, 4(Suppl I):SI 9

This article is available from: http://www.cancerci.com/content/4/SI/SI9

Allogeneic bone marrow cell (BMC) reconstitution of the non-myeloablatively conditioned host is supposed to provide an optimized platform for tumor vaccination. We recently showed that an allogeneic $\mathrm{T}$ cell-depleted graft was well accepted if the tumor-bearing host was NKdepleted. Based on this finding a vaccination protocol in tumor-bearing, non-myeloablatively conditioned, allogeneically reconstituted mice was elaborated. Vaccination was most efficient when allogeneically reconstituted, tumor-bearing mice received tumor-primed, donor $\mathrm{T}$ cells, which had matured in the allogeneic host together with host-derived tumor lysate-pulsed dendritic cells. High frequencies of tumor-specific proliferating and cytotoxic $\mathrm{T}$ cells were recorded, the survival time of tumorbearing mice was significantly prolonged and in over $50 \%$ of mice the tumor was completely rejected. Notably, GvH was not aggravated after vaccination with tumor-primed, donor-derived $\mathrm{T}$ cells that had matured in the host, i.e. T cells were tolerant towards the host, but not towards the tumor. The finding convincingly demonstrates the feasibility and efficacy of tumor vaccination of the allogeneically reconstituted, non-myeloablatively conditioned host after establishment of thymic tolerance and ceasing of initial GvH reactivities. Furthermore, they support the working hypothesis that reconstitution protocols favoring tolerance induction, which are time consuming, rather than a rapid establishment of full donor chimersim may allow more efficient tumor vaccination without potentially refreshing $\mathrm{GvH}$ reactivites. 